\title{
Pulmonary cavitary lesions may be one of the presenting features in $\lg$ A nephropathy
}

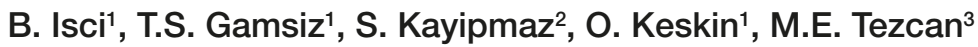 \\ ${ }^{1}$ Department of Internal Medicine, University of Health Sciences, Kartal Dr. Lutfi Kirdar Training \\ and Research Hospital, Istanbul, Turkey; ${ }^{2}$ Department of Pathology, University of Health Sciences, \\ Kartal Dr. Lutfi Kirdar Training and Research Hospital, Istanbul, Turkey; ${ }^{3}$ Department of Rheumatology, \\ University of Health Sciences, Kartal Dr. Lutfi Kirdar Training and Research Hospital, Istanbul, Turkey
}

\begin{abstract}
SUMMARY
Immunoglobulin A ( $\operatorname{Ig}$ A) nephropathy is the most frequent primary glomerulonephritis. Renal limited disease is the most widespread clinical form of the disease. Pulmonary involvement may also be seen concomitantly and capillaritis with pulmonary hemorrhage is the most frequent pulmonary involvement. In this paper, for the first time in literature, we describe an Ig A nephropathy patient with multiple pulmonary cavities as one of the presenting features of the disease. Also, no other etiology for the cavities was found other than Ig A nephropathy. Herein, possible pathogenesis might be capillaritis or deposition of immune complexes. As a result, it should be kept in mind that pulmonary cavity may be the presenting feature of Ig A nephropathy especially with other frequent signs of the disease.
\end{abstract}

Key words: Ig A nephropathy; Pulmonary renal syndrome; Pulmonary cavity.

\section{INTRODUCION}

Tmmunoglobulin A (Ig A) nephropathy is classically characterized by mesangial deposits of $\mathrm{Ig}$ A with asymptomatic microscopic hematuria or recurrent macroscopic hematuria (1). It may cause any form of glomerular pathology (2). Moreover, pulmonary involvement may also accompany Ig A nephropathy. The most common form of pulmonary pathology with Ig A nephropathy is alveolar capillaritis with pulmonary hemorrhage (3). Herein, we describe the first case in the literature that had pulmonary cavities associated with idiopathic IgA nephropathy.

\section{CASE REPORT}

A fifty-year-old male patient was admitted to the emergency department with complaints of macroscopic hematuria and cough. Before his admission, he had complaints of upper respiratory tract infection and he had taken non-steroidal anti-inflammatory drugs and amoxicillin clavulanate at the dose of $2 \mathrm{~g}$ daily for one week. At the time of admission, his physical examination revealed diminished breath sounds at the base of the left lung and basilar crackles in both hemithoraces. Moreover, the initial creatinine level of the patient was $7.1 \mathrm{mg} / \mathrm{dL}$. Analysis of a spot urine sample revealed proteinuria and microscopic hematuria. Microscopic urinalysis showed active urinary sediment. The spot urine protein/creatinine ratio also revealed subnephrotic proteinuria. Erythrocyte sedimentation rate and $\mathrm{C}$ reactive protein levels were $110 \mathrm{~mm} / \mathrm{h}$ and $120 \mathrm{mg} / \mathrm{L}$ respectively. We ordered thorax computerized tomography (CT) screening to investigate pulmonary symptoms. Pleural effusion in the left hemithorax and several cavitary lesions of $2 \mathrm{~mm}$ diameter in the right lung lower lobe posterobasal segment and the left lung lower lobe posterior segment were found on thorax CT (Figure 1). Furthermore, the complete blood count, other biochemical investigations and abdominal imaging performed to screen for underlying renal etiology
Corresponding author: Mehmet Engin Tezcan Department of Rheumatology Kartal Dr. Lutfi Kirdar Training and Research Hospital

34680 Kartal, Istanbul, Turkey E-mail: engintez@yahoo.com 


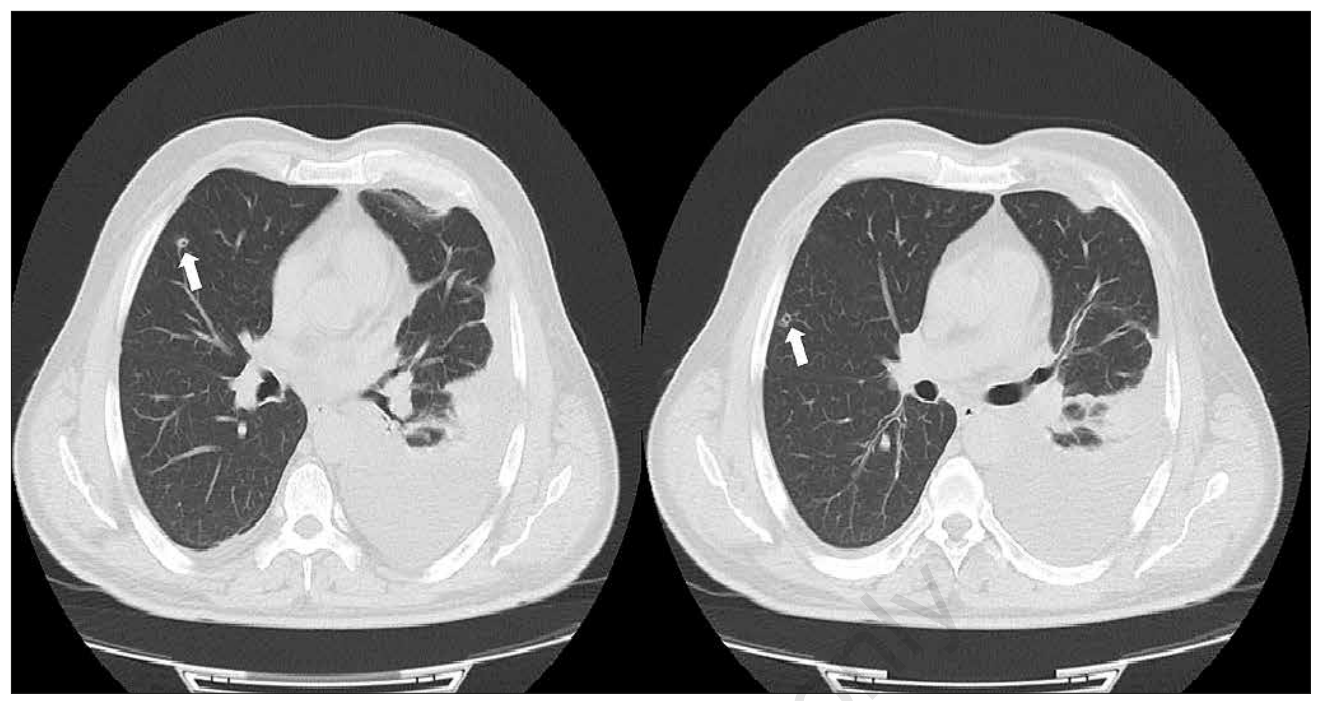

Figure 1 - Thorax computerized tomography images of the patient. The arrows show pulmonary cavitary lesions.

were found normal. The results of the serological tests, antinuclear antibodies, anti-neutrophilic cytoplasmic antibodies, anti-glomerular basement membrane antibodies were all negative. Complement levels were in the normal range. Both acid fast bacillus testing and tuberculosis culture were negative in sputum. Also, a puri-

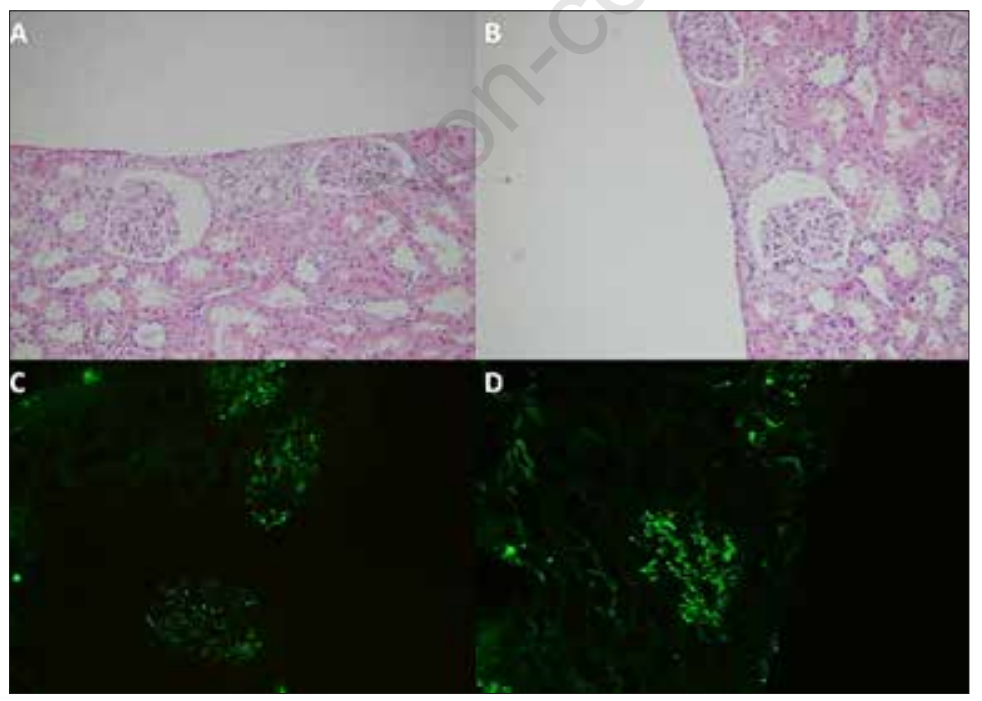

Figure 2 - Kidney biopsy specimens with hematoxylin eosin staining and x200 magnification that show mesangial proliferation in the glomeruli (A and B). Kidney biopsy specimens with immunofluorescence staining that show $\lg A$ deposits $(C)$ and complementary 3 aggregation (D). fied protein derivative skin test was found $<5 \mathrm{~mm}$. Bacterial and fungus cultures of sputum were also negative. Additionally, procalcitonin levels were found at $<0.1$ $\mathrm{ng} / \mathrm{ml}$. Transthoracic echocardiography for investigating infective endocarditis was normal. Also, all random blood cultures were negative. Then, we conducted a kidney biopsy. It was consistent with IgA nephropathy without crescents and any sign of vasculitis (Figure 2). We planned to make fiberoptic bronchoscopy to take a lung biopsy and bronchoalveolar lavage in order to investigate the pulmonary pathology. However, the patient could not tolerate the procedure. Therefore, we ordered sputum cytology to exclude primary pulmonary malignancy. Non-specific inflammation was reported in sputum cytology. So, we could not exclude infection and malignancy more accurately with fiberoptic bronchoscopy.

With these findings, the patient was considered to have IgA nephropathy triggered by infection and associated pulmonary cavitation. At the time of admission, the patient was hemodialysed. Pulse intravenous methylprednisolone at a dose of $1 \mathrm{~g}$ daily for three consecutive days followed by prednisolone at a dose of $1 \mathrm{mg} / \mathrm{kg} /$ day and monthly cyclophosphamide therapy was 
started. At following visits, with immunosuppressive treatments, his renal function returned to normal levels. Furthermore, the number and the size of the cavities were found decreased and pleural effusion was resolved in follow-up imaging.

\section{DISCUSSION}

IgA nephropathy is the most common form of primary glomerulonephritis (2). The clinical findings usually emerge following an infection. The disease usually has a benign course and is a renal limited disease. But, It may cause progressive kidney disease with $25-30 \%$ of patients developing end-stage renal disease within 20 years of diagnosis (4). Furthermore, rapidly progressive glomerulonephritis develops in about $5 \%$ of the patients (5). Pulmonary involvements might also be observed. Ig A nephropathy rarely presents as concomitant renal and pulmonary findings. The most common reported form of pulmonary involvement is pulmonary hemorrhage with probable alveolar capillaritis $(3,6)$. As far as we know there is no patient with IgA nephropathy in literature that has pulmonary cavitary lesions as one of the presenting features.

Concomitant pulmonary and renal findings may be seen in several auto-immune diseases. The most frequent causes are Goodpasture syndrome, systemic lupus erythematosus, granulomatosis with polyangiitis and microscopic polyangiitis (7). However, in our patient, there was no any sign of it being either clinically or serologically related to any specific autoimmune disease. Furthermore, infectious diseases and malignancies may cause pulmonary cavities. We excluded tuberculosis and other specific bacterial and fungal infections with both microbiological exams and procalcitonin test. We could only exclude malignancy with sputum cytology. In this case, we could take a tissue sample only from the kidney due to the patient's clinical situation. Even though he needed renal replacement treatment in the first days of hospitalization, the kidney biopsy was compatible with Ig
A nephropathy without features of rapidly progressive glomerulonephritis. Additionally, in our patient, as expected in Ig A nephropathy, his symptoms were triggered by upper respiratory tract infection. Even though we could not exclude infection and malignancy with fiberoptic bronchoscopy, the success of the therapy might support our clinical evaluation. Moreover, Ig A vasculitis may also cause Ig A nephropathy with severe systemic involvement including pulmonary hemorrhage. However, there was no feature related to vasculitis in his renal biopsy. Also, there were no other concomitant systemic features except pulmonary cavities from which we were able to obtain a tissue sample to show vasculitis. In this case, the etiology of pleural effusion might be endothelial damage produced by inflammatory mechanisms increasing capillary permeability. Effusion was also ameliorated after treatment. So we diagnosed him with possible idiopathic Ig A nephropathy and accompanying pulmonary cavities probably related with Ig A nephropathy.

There is no proven pathogenesis for pulmonary involvement in IgA nephropathy. Nonspecific Ig A immune-response against alveolar capillaries after upper respiratory tract infection might be the first possible mechanism for pulmonary injury (8). Capillaritis is the most common pathogenesis for Ig A related pulmonary injuries. Pulmonary hemorrhage usually follows the capillaritis. In these hypotheses, direct interaction between capillary and Ig A antibodies may cause injury (3). The second possible mechanism is the deposition of immune complexes with Ig A as in kidney mesangium (9). These immune complexes have high affinity for the extracellular-matrix components fibronectin and type IV collagen in the mesangium. They usually bind and activate mesangium cells. Then they activate the complement system in alveoli. Hence, the complementary system augments the inflammatory cascade and potentiates tissue injury (10). Furthermore, immune complexes with Ig A may also cause capillaritis instead of mesangium immune complex interaction (6). 


\section{CONCLUSIONS}

Although pulmonary hemorrhage is the most frequent pulmonary involvement in Ig A nephropathy, pulmonary cavitary lesions may also accompany the disease as one of the presenting feature. In so far as Ig A nephropathy usually emerges after infectious diseases and may be secondary to other auto-immune diseases, the first thing to be done should be to exclude infectious diseases with detailed diagnostic procedures and to investigate accompanying auto-immune diseases.

\section{REFERENCES}

1. Wyatt RJ, Julian BA. IgA nephropathy. N Engl J Med. 2013; 368: 2402-14.

2. Floege J, Amann K. Primary glomerulonephritides. Lancet. 2016; 387: 2036-48.

3. Green RJ, Ruoss SJ, Kraft SA, et al. Pulmo- nary capillaritis and alveolar hemorrhage. Update on diagnosis and management. Chest. 1996; 110: 1305-16.

4. Barratt J, Feehally J. Treatment of IgA nephropathy. Kidney Int. 2006; 69: 1934-8.

5. Jennette JC. Rapidly progressive crescentic glomerulonephritis. Kidney Int. 2003; 63: 1164-77.

6. Oluwole K, Esuzor L, Adebiyi O, et al. Pulmonary hemorrhage with hematuria: do not forget IgA nephropathy. Clin Kidney J. 2012; 5: 463-6.

7. Brusselle GG. Pulmonary-renal syndromes. Acta Clin Belg. 2007; 62: 88-96.

8. Rambausek M, Ritz E, Waldherr R. Recurrent bloody dialysate during upper respiratory tract infection in mesangial IgA glomerulonephritis. Nephron. 1987; 46: 213.

9. Anantham D, Chan KP, Chuah KL, et al. Pulmonary capillaritis in IgA nephropathy. Southern Med J. 2007; 100: 605-7.

10. Suzuki H, Kiryluk K, Novak J, et al. The pathophysiology of IgA nephropathy. J Am Soc Nephrol. 2011; 22: 1795-803. 